\title{
Quantum Footprints of Symplectic Rigidity
}

\author{
Leonid Polterovich (Tel Aviv University, Tel Aviv, Israel)
}

Suddenly the result turned out completely different from what he had expected: again it was $1+1=2$. "Wait a minute!" he cried out, "Something's wrong here." And at that very moment, the entire class began whispering the solution to him in unison: "Planck's constant! Planck's constant!"

After M. Pavic, Landscape Painted with Tea, 1988

In this note, we discuss an interaction between symplectic topology and quantum mechanics. The interaction goes in both directions. On one hand, ideas from quantum mechanics give rise to new notions and structures on the symplectic side and, furthermore, quantum mechanical insights lead to useful symplectic predictions when topological intuition fails. On the other hand, some phenomena discovered within symplectic topology admit a meaningful translation into the language of quantum mechanics, thus revealing quantum footprints of symplectic rigidity.

\section{What is ... symplectic?}

A symplectic manifold is an even-dimensional manifold $M^{2 n}$ equipped with a closed differential 2-form $\omega$ that can be written as $\sum_{i=1}^{n} d p_{i} \wedge d q_{i}$ in appropriate local coordinates $(p, q)$. For an oriented surface $\Sigma \subset M$, the integral $\int_{\Sigma} \omega$ plays the role of a generalised area, which, in contrast to the Riemannian area, can be negative or vanish.

To have some interesting examples in mind, think of surfaces with an area form and their products, as well as complex projective spaces equipped with the Fubini-Study form, and their complex submanifolds.

Symplectic manifolds model the phase spaces of systems of classical mechanics. Observables (i.e. physical quantities such as energy, momentum, etc.) are represented by functions on $M$. The states of the system are encoded by Borel probability measures $\mu$ on $M$. The simplest states are given by the Dirac measure $\delta_{z}$ concentrated at a point $z \in M$.

The laws of motion are governed by the Poisson bracket, a canonical operation on smooth functions on $M$, given by $\{f, g\}=\sum_{j}\left(\frac{\partial f}{\partial q_{j}} \frac{\partial g}{\partial p_{j}}-\frac{\partial f}{\partial p_{j}} \frac{\partial g}{\partial q_{j}}\right)$. The evolution of the system is determined by its energy, a time-dependent function $f_{t}$ : $M \rightarrow \mathbb{R}$ called its Hamiltonian. Hamilton's famous equation describing the motion of a system is given, in the Heisenberg picture, by $\dot{g}_{t}=\left\{f_{t}, g_{t}\right\}$, where $g_{t}=g \circ \phi_{t}^{-1}$ stands for the time evolution of an observable function $g$ on $M$ under the Hamiltonian flow $\phi_{t}$. The maps $\phi_{t}$ are called Hamiltonian diffeomorphisms. They preserve the symplectic form $\omega$ and constitute a group with respect to composition.
In the 1980s, new methods, such as Gromov's theory of pseudo-holomorphic curves and the Floer-Morse theory on loop spaces, gave birth to "hard" symplectic topology. It detected surprising symplectic rigidity phenomena involving symplectic manifolds, their subsets and diffeomorphisms. A number of recent advances show that there is yet another manifestation of symplectic rigidity taking place in function spaces associated to a symplectic manifold. Its study forms the subject of function theory on symplectic manifolds, a rapidly evolving area whose development has led to the interactions with quantum mechanics described below.

\section{The non-displaceable fiber theorem}

In 1990, Hofer [21] introduced an intrinsic "small scale" on a symplectic manifold: a subset $X \subset M$ is called displaceable if there exists a Hamiltonian diffeomorphism $\phi$ such that $\phi(X) \cap$ $X=\emptyset$.

Let us illustrate this notion in the case when $M=S^{2}$ is the two-dimensional sphere equipped with the standard area form. Any disc lying in the upper hemisphere is displaceable: one can send it to the lower hemisphere by a rotation. However, the equator (a simple closed curve splitting the sphere into two discs of equal area) is non-displaceable by any areapreserving transformation (see Figure 1). This example demonstrates the contrast between symplectic "smallness" and measure theoretic "smallness": the equator has measure 0 , yet it is large from the viewpoint of symplectic topology.

The central result discussed in this note brings together (non)-displaceability and Poisson commutativity.

Theorem 2.1 (Non-displaceable fiber theorem, [12]). Let $\vec{f}=\left(f_{1}, \ldots, f_{N}\right): M \rightarrow \mathbb{R}^{N}$ be a smooth map of a closed symplectic manifold $M$ whose coordinate functions $f_{i}$ pairwise Poisson commute: $\left\{f_{i}, f_{j}\right\}=0$. Then, $\vec{f}$ possesses a nondisplaceable fiber: for some $w \in \mathbb{R}^{N}$, the set $\vec{f}^{-1}(w)$ is nonempty and non-displaceable.
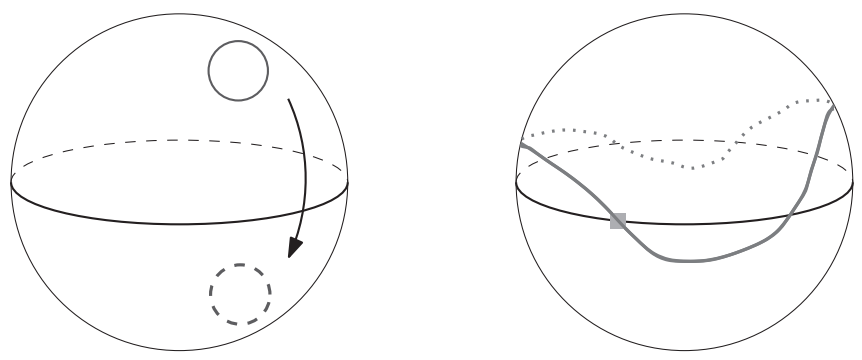

Figure 1. (Non)-displaceability on the sphere 
Maps with pairwise commuting components naturally arise in the theory of integrable systems and in Hamiltonian tori actions (the moment maps). Roughly speaking, the theorem above states that each such map necessarily possesses a symplectically large fiber. Let us note that interesting classes of maps that necessarily possess "large" fibers appear in several seemingly remote areas of mathematics, from combinatorics to Riemannian geometry [20]. It would be interesting to explore this analogy.

Detecting non-displaceability of subsets of symplectic manifolds is a classical problem going back to Arnold's seminal Lagrangian intersections conjecture. In fact, the very existence of subsets that can be displaced by a volume preserving diffeomorphism but not by a Hamiltonian one is a manifestation of symplectic rigidity. Theorem 2.1 provides a useful tool in the following situation. Assume that we know a priori that all but possibly one fiber of a map $\vec{f}: M \rightarrow \mathbb{R}^{N}$ with Poisson-commuting components are displaceable. Then that particular fiber is necessarily non-displaceable.

As an illustration, assume that $M=S^{2} \times S^{2}$ is the product of two spheres, and $\vec{f}=\left(z_{1}, z_{2}\right)$, where $z_{i}$ is the height function on the $i$-th copy of $S^{2}$. One can see that all the fibers, except possibly the Clifford torus $L$ given by the product of the equators, are displaceable (see Figure 2). Therefore, $L$ is non-displaceable (see [29]).

The non-displaceable fiber theorem has the following equivalent formulation.

Theorem 2.2 (Rigidity of partitions of unity, [13]). A finite open cover of a closed symplectic manifold by open displaceable sets does not admit a Poisson-commuting partition of unity.

Interestingly enough, both the proof and the interpretation of this result involve quantum mechanics.
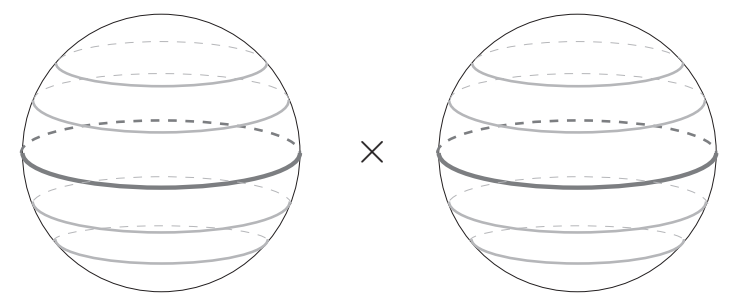

Figure 2. The Clifford torus

\section{$3 \quad$ Encountering quantum mechanics}

The mathematical model of quantum mechanics starts with a complex Hilbert space $H$. In what follows, we shall focus on finite-dimensional Hilbert spaces only, as they are quantum counterparts of closed symplectic manifolds. Observables are represented by Hermitian operators whose space is denoted by $\mathcal{L}(H)$. The states are provided by density operators, i.e. positive trace-one operators $\rho \in \mathcal{L}(H)$. Pure states are given by rank-one projectors. They are usually identified with the unit vectors in $H$ generating their images and defined up to a phase factor.

Quantization is a formalism behind quantum-classical correspondence, a fundamental principle stating that quantum
Table 1. Quantum-classical correspondence

\begin{tabular}{lcc}
\hline & Classical & Quantum \\
\hline & Symplectic manifold $(M, \omega)$ & $\mathbb{C}$-Hilbert space $H$ \\
Observables & $f \in C^{\infty}(M)$ & $T_{\hbar}(f) \in \mathcal{L}(H)$ \\
States & Probability measures on $M$ & $\rho \in \mathcal{L}(H), \rho \geq 0, \operatorname{trace}(\rho)=1$ \\
Bracket & $\{f, g\}$ & $-(i / \hbar)\left[T_{\hbar}(f), T_{\hbar}(g)\right]$ \\
\hline
\end{tabular}

mechanics contains the classical one as a limiting case when the Planck constant $\hbar \rightarrow 0$. Mathematically, the correspondence in question is a linear map $f \mapsto T_{\hbar}(f)$ between smooth functions on a symplectic manifold and Hermitian linear operators on a complex Hilbert space $H$ depending on $\hbar$. The dimension of $H$ grows to $\infty$ as $\hbar \rightarrow 0$. The map $T_{\hbar}$ is assumed to satisfy a number of axioms, some of which are summarised in Table 1. Let us emphasise that the quantum-classical correspondence is not precise. It holds true up to an error that is small with $\hbar$.

In finite-dimensional quantum mechanics, observables take a finite number of values only. Given an observable $A$, let $A=\sum_{i=1}^{k} \lambda_{i} P_{i}$ be its spectral decomposition, where the $P_{i}$ 's are pairwise orthogonal projectors. According to the main statistical postulate, in a state $\rho$ the observable $A$ takes the values $\lambda_{i}$ with probabilities trace $\left(P_{i} \rho\right)$.

The finiteness is well illustrated by the famous SternGerlach experiment dealing with the deflection of a beam of atoms passing through a specially chosen magnetic field. This experiment highlighted the following phenomenon: the vertical component of the angular momentum of the atoms takes only two values \pm 1 , as opposed to the classical prediction that its values fill the interval $[-1,1]$. Recall that the angular momentum $L=\left(L_{1}, L_{2}, L_{3}\right)$ is an attribute of a rotating body depending on its angular velocity and shape. Its phase space is the unit sphere $S^{2} \subset \mathbb{R}^{3}$. Its components satisfy the commutation relation $\left\{L_{1}, L_{2}\right\}=L_{3}$ and its cyclic permutations. In quantum mechanics, the components of the angular momentum correspond to $2 \times 2$ Pauli matrices whose commutator relations are (up to a factor) the same as of $L_{i}$ 's and whose eigenvalues are \pm 1 . This perfectly explains the SternGerlach phenomenon. However, we immediately arrive at the following paradox [33]. Look (on the quantum side) at the projections of $L$ on three unit vectors $u, v$ and $w$ in $\mathbb{R}^{3}$, with $u+v+w=0$. By symmetry considerations, each of these projections also takes a value \pm 1 , while their sum equals 0 , and we get a contradiction! One of the resolutions of this paradox is as follows: the quantum-classical correspondence takes these projections to pairwise non-commuting matrices, hence we cannot measure them simultaneously. In quantum mechanics, we face a new role of the bracket: it controls uncertainty. According to the Heisenberg uncertainty principle, for every pair of observables $A, B \in \mathcal{L}(H)$ and a state $\rho$,

$$
\operatorname{Variance}(A, \rho) \cdot \operatorname{Variance}(B, \rho) \geq \frac{1}{4} \cdot|\operatorname{trace}([A, B] \cdot \rho)|^{2} .
$$

\section{$4 \quad$ From quantum indeterminism to quasi-states}

In his foundational 1932 book [42], von Neumann defined quantum states as real valued functionals $\rho: \mathcal{L}(H) \rightarrow \mathbb{R}$ satisfying three simple axioms: $\rho(\mathbb{1})=1$ (normalization), 


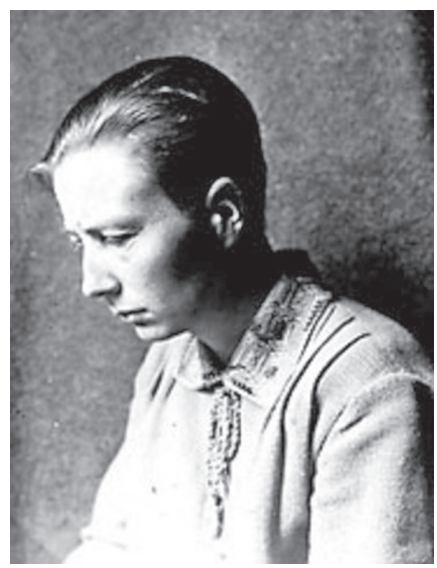

Figure 3. Grete Hermann (1901-1984)

$\rho(A) \geq 0$ if $A \geq 0$ (positivity) and linearity. Next, he showed that each such functional can be written as $\rho(A):=\operatorname{trace}(\rho A)$, where $\rho$ is a density operator. Interpreting $\rho(A)$ as the expectation of the observable $A$ in the state $\rho$, von Neumann concluded that for any quantum state $\rho$, there exists an observable $A$ such that the variance $\rho\left(A^{2}\right)-\rho(A)^{2}$ is strictly positive. In other words, in sharp contrast to Dirac $\delta$-measures in classical mechanics, there are no quantum states in which the values of all observables are deterministic.

This conclusion, known as the impossibility to introduce hidden variables in quantum mechanics, caused a passionate discussion among physicists. According to Grete Hermann (1935), a German physicist and philosopher, the linearity axiom only makes sense for observables $A, B$ that can be measured simultaneously, i.e. that commute: $[A, B]=0$. It should be mentioned that Hermann's criticism went unnoticed for almost three decades until the work of Bell, perhaps because the times were tough and Hermann had no opportunity to properly advertise her ideas as she became active in the underground movement against the Nazis. An account of this story is given in a lovely book by L. Gilder [16]; one could also see the Wikipedia article about Grete Hermann (from where the photo in Figure 3 has been sourced) and references therein. An attempt to incorporate Hermann's criticism leads to the following definition. A quantum quasi-state is a functional $\rho: \mathcal{L}(H) \rightarrow \mathbb{R}$ that satisfies the normalization and positivity axioms, while linearity is relaxed as follows: $\rho$ is linear on every commutative subspace of $\mathcal{L}(H)$ (quasi-linearity).

However, in 1957, Gleason proved the following remarkable theorem. If $H$ has complex dimension 3 or greater, any quantum quasi-state is linear, that is, it is a quantum state. This confirms Neumann's conclusion. Citing Peres [33, p. 196], "Gleason's theorem is a powerful argument against the hypothesis that the stochastic behavior of quantum tests can be explained by the existence of a subquantum world, endowed with hidden variables whose values unambiguously determine the outcome of each test."

Let us now mimic the definition of a quantum quasi-state in classical mechanics, using the quantum-classical correspondence and keeping in mind that commuting Hermitian operators correspond to Poisson-commuting functions. Let $(M, \omega)$ be a closed symplectic manifold. A symplectic quasistate on $M$ is a functional $\zeta: C(M) \rightarrow \mathbb{R}$ such that $\zeta(1)=1$ (normalization), $\zeta(f) \geq 0$ for $f \geq 0$ (positivity) and $\zeta$ is linear on every Poisson-commutative subspace (quasi-linearity).

In contrast to quantum mechanics, certain symplectic manifolds admit nonlinear symplectic quasi-states. Starting from [12], this "anti-Gleason phenomenon" in classical mechanics has been established for various manifolds, including complex projective spaces and their products, toric manifolds, blow ups and coadjoint orbits [32, 40, 15, 6].

In terms of the existence mechanism for symplectic quasistates, there is a mysterious dichotomy (vaguely resembling the rank-one versus higher rank dichotomy in Lie theory). In dimension 2 (i.e. for surfaces), symplectic quasi-states exist in abundance. Their construction is provided by the theory of topological quasi-states by Aarnes [1], whose motivation was to explore the validity of the Gleason theorem for algebras of functions on topological spaces, where the quasi-linearity is understood as linearity on all singly-generated subalgebras. In fact, in dimension 2, topological and symplectic quasi-states coincide. However, all known nonlinear symplectic quasistates in higher dimensions come from Floer theory.

Interestingly enough, Floer-theory quasi-states come with a trove of additional features, which make them useful for various applications in symplectic topology. In particular, $\zeta(f)=0$ for every function $f$ with displaceable support (vanishing property). This immediately yields the rigidity of partitions of unity, which in turn is equivalent to the nondisplaceable fiber theorem (see Theorems 2.2 and 2.1 above). Indeed, assume that $f_{1}, \ldots, f_{N}$ are pairwise commuting functions with displaceable supports that sum to 1 . By the vanishing property, $\zeta\left(f_{i}\right)=0$. By normalization and quasi-linearity, $1=\zeta\left(\sum f_{i}\right)=\sum \zeta\left(f_{i}\right)=0$, and we get a contradiction.

\section{Quasi-states from Floer theory}

Here, the reader is invited to catch a glimpse of Floer theory, with a focus on the construction of symplectic quasi-states. To this end, it is time to reveal the main secret of "hard" symplectic topology: the actual object of study is not the symplectic manifold itself but the space $L M$ of all contractible loops $z: S^{1} \rightarrow M$. The symplectic structure $\omega$ induces a functional $\mathcal{A}: L M \rightarrow \mathbb{R}$ as follows. Given a loop $z$, take any disc $D \subset M$ spanning $z$ (see Figure 4) and put $\mathcal{A}(z)=-\int_{D} \omega$. Since $\omega$ is a closed form, the integral does not change under homotopies of the disc with fixed boundary and, therefore, $\mathcal{A}$ is well defined up to the homotopy class of $D$, an ambiguity we shall ignore. Its critical points are degenerate: they form the submanifold of all constant loops. In order to resolve this degeneracy, fix a time-dependent Hamiltonian $f_{t}: M \rightarrow \mathbb{R}$, $t \in S^{1}$, and define a perturbation $\mathcal{A}_{f}: L M \rightarrow \mathbb{R}$ of $\mathcal{A}$ by

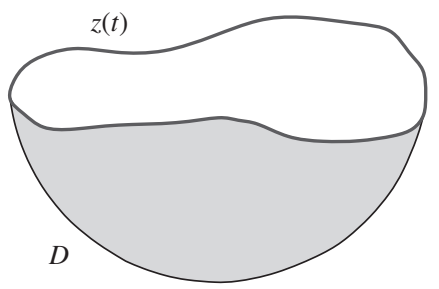

Figure 4. A disc spanning a loop 


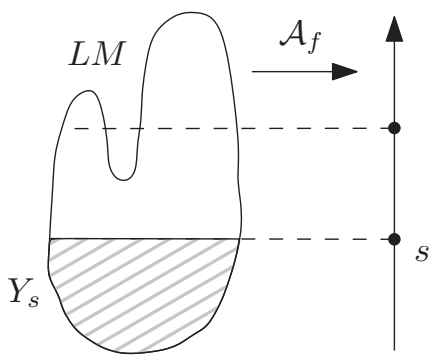

Figure 5. A topological "phase transition"

$\mathcal{A}_{f}(z)=\mathcal{A}(z)+\int_{0}^{1} f_{t}(z(t)) d t$. This is the classical action functional. Ironically, the perturbations become the main object of interest.

Roughly speaking, Floer theory is the Morse theory for $\mathcal{A}_{f}$. The space $L M$ carries a special class of Riemannian metrics associated to almost complex structures on $M$. Pick such a metric and look at the space of the gradient trajectories of $\mathcal{A}_{f}$ connecting two critical points (i.e. two periodic orbits). Note that in $M$ such a trajectory is a path of loops, i.e. a cylinder. It was a great insight of Floer [14] that these cylinders satisfy a version of the Cauchy-Riemann equation with asymptotic boundary conditions and, in particular, they can be studied within Gromov's theory of pseudo-holomorphic curves [18]. Even though the gradient flow of $\mathcal{A}_{f}$ is ill-defined, this asymptotic boundary problem is well posed and Fredholm. With this key ingredient at hand, one can build a meaningful version of the Morse theory of the action functional on the loop space. It is powerful enough to detect topological "phase transitions" of the sublevel sets $Y_{s}:=\left\{\mathcal{A}_{f}<s\right\}$ as $s$ runs from $+\infty$ to $-\infty$ (see Figure 5). They happen at special critical values $s=c(f)$ of $\mathcal{A}_{f}$, called spectral invariants, which were discovered and studied by C. Viterbo [41], M. Schwartz [38] and Y.-G. Oh [30] (also see [28, 36, 31]). The symplectic quasi-state $\zeta$ introduced in [12] captures such transitions for high energies: $\zeta(f):=\lim _{E \rightarrow+\infty} c(E f) / E$.

\section{$6 \quad$ An example: The median quasi-state}

In general, Floer-homological quasi-states do not admit a simple description. However, there is one exception. First, we define a quasi-state $\zeta: C\left(S^{2}\right) \rightarrow \mathbb{R}$ on smooth Morse functions $f \in C^{\infty}\left(S^{2}\right)$, where the sphere $S^{2}$ is equipped with the area form $\omega$ of total area 1. Recall that the Reeb graph $\Gamma$ of $f$ is obtained from $S^{2}$ by collapsing connected components of the level sets of $f$ to points (see Figure 6). In the case of $S^{2}$, the Reeb graph is necessarily a tree. Denote the natural projection by $\pi: S^{2} \rightarrow \Gamma$. The push-forward of the symplectic area on the sphere is a probability measure on $\Gamma$. It is not hard to show

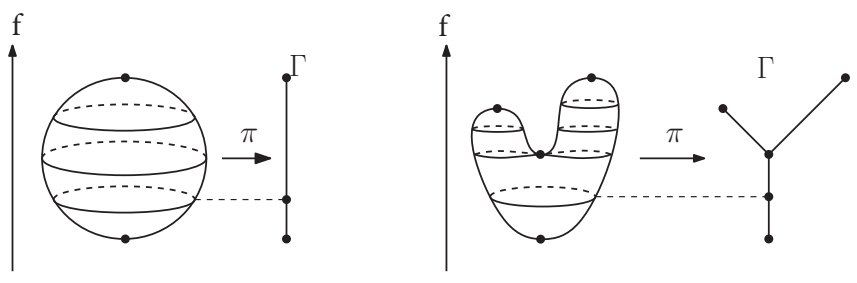

Figure 6. The Reeb graph (and, in fact, it is well known in combinatorial optimisation) that there exists a unique point $m \in \Gamma$, called the median of $\Gamma$, such that each connected component of $\Gamma \backslash\{m\}$ has measure $\leq \frac{1}{2}$ (see [11, Section 5.3]). Define $\zeta(f)$ as the value of $f$ on the level $\pi^{-1}(m)$. It turns out that $\zeta$ is Lipshitz in the uniform norm and its extension to $C(M)$ is a nonlinear quasi-state the one which comes from Floer theory on $S^{2}$.

\section{$7 \quad$ Noise-localization uncertainty}

Recall that a finite open cover of a closed symplectic manifold by open displaceable sets does not admit a Poissoncommuting partition of unity. Can the functions entering the partition of unity "almost commute"? It turns out that the rigidity of partitions of unity phenomenon admits the following quantitative version. Throughout this section, we fix a finite cover $\mathcal{U}=\left\{U_{1}, \ldots, U_{N}\right\}$ of $M$ by open displaceable sets.

For a finite collection $\vec{f}=\left(f_{1}, \ldots, f_{N}\right)$ of smooth functions on $M$, define the quantity

$$
v(\vec{f})=\max _{x, y \in[-1,1]^{N}}\left\|\left\{\sum_{i} x_{i} f_{i}, \sum_{i} y_{i} f_{i}\right\}\right\|,
$$

which measures the magnitude of non-commutativity of these functions. Here $\|\cdot\|$ stands for the uniform norm.

Now introduce the Poisson bracket invariant [34] of the cover $\mathcal{U}$ :

$$
\operatorname{pb}(\mathcal{U})=\inf _{\vec{f}} v(\vec{f}),
$$

where the infimum is taken over all partitions of unity subordinated to $\mathcal{U}$. It measures the minimal possible magnitude of non-commutativity of a partition of unity subordinated to $\mathcal{U}$.

Next, define the symplectic size $\operatorname{Size}(U)$ of a displaceable subset $U \subset M$ as the minimal time $T$ needed in order to displace $U$ with unit energy, i.e. by using a Hamiltonian $f_{t}$, $t \in[0, T]$, with $\left\|f_{t}\right\|=1$ for all $t$. The size is a fundamental symplectic invariant (which is usually called the displacement energy) introduced by Hofer [21]. It is an important fact, proved in full generality by Lalonde and McDuff [24], that the size of a ball of sufficiently small radius $r$ in $M$ is $\sim r^{2}$. Define the size of the cover $\mathcal{U}$ by $\operatorname{Size}(\mathcal{U}):=\max _{i} \operatorname{Size}\left(U_{i}\right)$.

It turns out that

$$
\operatorname{pb}(\mathcal{U}) \cdot \operatorname{Size}(\mathcal{U}) \geq C>0,
$$

where the constant $C$ depends, roughly speaking, on the local geometry and combinatorics of the cover. We refer to works by the author [34], Seyfaddini [39] and Ishikawa [22] for more information about this constant.

An interpretation of this result comes from the phase localization problem in quantum mechanics. Here we think of the cover $\mathcal{U}=\left\{U_{1}, \ldots, U_{N}\right\}$ as a small scale coarse-graining of $M$. Given a particle $z$ on $M$, we wish to localize it in the phase space, i.e. to provide an answer to the following question: to which of the sets $U_{i}$ does $z$ belong? Of course, the question is ambiguous due to overlaps between the sets $U_{i}$. Following an idea of I. Polterovich, we illustrate this by a toy model of a cellular communication network consisting of a collection of access points $u_{1}, \ldots, u_{N}$. Each access point $u_{j}$ can be reached from a domain $U_{j}$, the so-called location area. The location areas $U_{j}$ cover some territory $M$ (e.g. Europe). Your phone at a given location $z \in M$ must register in exactly one access point $u_{j}$, whose location area $U_{j}$ contains $z$ (see Figure 7). 


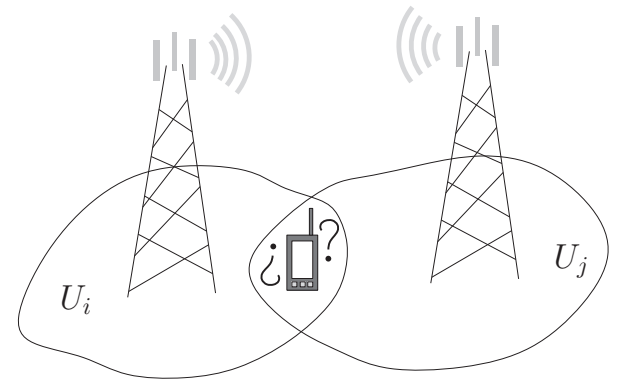

Figure 7. The registration procedure

In order to resolve the ambiguity, let us make the required assignment $z \mapsto U_{j}$ at random: fix a partition of unity $\vec{f}=\left(f_{1}, \ldots, f_{N}\right)$ subordinated to $\mathcal{U}$ and register $z$ in $U_{j}$ with probability $f_{j}(z)$. Since $f_{j}$ is supported in $U_{j}$, this procedure provides "the truth, but not the whole truth".

Now, consider the quantum version of the registration procedure. Let us assume that the quantum-classical correspondence takes a function $f_{j}$ to a positive Hermitian operator $A_{j}$ on a Hilbert space $H$. This holds, for instance, in the framework of Berezin-Toeplitz quantization [2, 3, 19, 4, 25, 7], (see [37] for a beautiful survey). With this assumption at hand, in a state $\rho$ the probability of registration of the particle in the set $U_{j}$ equals trace $\left(T_{\hbar}\left(f_{j}\right) \rho\right)$.

The quantum registration procedure exhibits noise ("an increment of variances"), which is governed by the operator norms of the commutators $\left\|\left[A_{i}, A_{j}\right]\right\|_{o p} \approx \hbar\left\|\left\{f_{i}, f_{j}\right\}\right\|$. The noise and the symplectic size turn out to be related by the following noise-localization uncertainty relation [34]:

$$
\text { Noise } \times \operatorname{Size}(\mathcal{U}) \geq C \hbar,
$$

where the constant $C$ is related to the one in (1). Its physical meaning fits the uncertainty principle: a sufficiently fine localization yields a large noise. In fact, inequality (1) was discovered after a translation into quantum language: a purely symplectic intuition yields a much worse lower bound.

We refer to a survey by Bush, Lahti and Werner [5] for a discussion of noise in quantum measurements, and to Kalai's article [23] for an intriguing link between quantum noise production and non-commutativity in the context of quantum computing.

\section{$8 \quad$ Classical vs. quantum speed limit}

How long does it take to displace with unit energy a state concentrated in a subset $U$ of the phase space? In the classical framework, this speed limit is governed by the symplectic size introduced above.

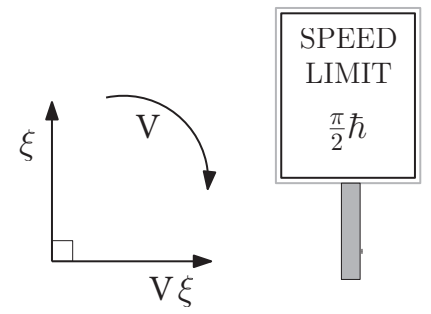

Figure 8. "Displacing" a pure quantum state
In the quantum world, the dynamics of a system with (time-dependent) energy $F_{t} \in \mathcal{L}(H)$ are described by the unitary evolution $V_{t}: H \rightarrow H$ satisfying the Schrödinger equation $\dot{V}_{t}=-(i / \hbar) F_{t} V_{t}$. The displacement (at least, for pure states $\xi \in H,|\xi|=1$ ) corresponds to orthogonalisation: a unitary transformation $V$ displaces $\xi$ if $\langle V \xi, \xi\rangle=0$ (see Figure 8). Interestingly enough, the universal quantum speed limit was discovered by the physicists Mandelstam and Tamm [26] as early as 1945 and refined by Margolus and Levitin [27] in 1998. It turns out that the minimal possible orthogonalisation time with unit energy, i.e. with $\left\|F_{t}\right\|_{o p}=1$ for all $t$, equals $(\pi / 2) \hbar$. Thus (carrying out reverse engineering of the past in the spirit of the Ministry of Truth), one can argue that the definition of symplectic size could have been found over four decades before Hofer if somebody would have taken the trouble to dequantize it!

On a more serious note, in a recent work with Charles [9], we addressed a question about semiclassical "displacement" of semiclassical states. It turns out that if such a state is concentrated in a ball of radius $\sim \hbar^{\epsilon}, \epsilon \in[0,1 / 2)$, the minimal displacement time is $\sim \hbar^{2 \epsilon}$. Thus, on the scale exceeding the quantum length scale $\sim \sqrt{\hbar}$, the semiclassical speed limit is more restrictive. The proofs involve both symplectic topology and semiclassical analysis and, in particular, the sharp remainder estimates for Berezin-Toeplitz quantization found in [8].

\section{$9 \quad$ Epilogue}

Nowadays, symplectic geometry is a well developed subject. Its many facets include, in particular, "hard" symplectic topology (notably, Floer theory) and quantization. In this note, we have described some first steps toward understanding the interactions between these areas, which highlight quantum mechanics as a playground for testing and applications of "hard" symplectic methods. In general, a meaningful translation of symplectic rigidity phenomena involving subsets and diffeomorphisms into the language of quantum mechanics faces serious analytical and conceptual difficulties. However, such a translation becomes possible if one shifts the focus from subsets and morphisms of manifolds to function spaces. The latter exhibit interesting structures and features such as symplectic quasi-states and rigidity of partitions of unity, which are systematically studied within function theory on symplectic manifolds.

\section{Acknowledgments.}

In parts, the results surveyed above are in partnership with Laurent Charles, Michael Entov and Frol Zapolsky; I cordially thank them all. I am very grateful to Karina Samvelyan for the skilful preparation of the figures and useful comments, as well as to Andrei Iacob for superb copyediting. This note is an abridged version of my paper [35]. This research was partially supported by the Israel Science Foundation grant 178/13 and the European Research Council Advanced grant 338809.

\section{References}

[1] J. Aarnes, Quasi-states and quasi-measures. Adv. Math. 86 (1991), 41-67. 
[2] F. Berezin, General concept of quantization. Comm. Math. Phys. 40 (1975), 153-174.

[3] M. Bordemann, E. Meinrenken and M. Schlichenmaier, Toeplitz quantization of Kähler manifolds and $g l(N), N \rightarrow \infty$ limits. Comm. Math. Phys. 165 (1994), 281-296.

[4] D. Borthwick and A. Uribe, Almost complex structures and geometric quantization. Math. Res. Lett. 3 (1996), 845-861.

[5] P. Busch, P. Lahti and R. Werner, Colloquium: Quantum rootmean-square error and measurement uncertainty relations. Reviews of Modern Physics 86 (2014): 1261.

[6] A. Caviedes Castro, Calabi quasimorphisms for monotone coadjoint orbits. arXiv:1507.06511, to appear in the Journal of Topology and Analysis.

[7] L. Charles, Quantization of compact symplectic manifolds, Journal of Geometric Analysis 26 (2016), 2664-2710.

[8] L. Charles and L. Polterovich, Sharp correspondence principle and quantum measurements, arXiv:1510.02450, Preprint, 2015.

[9] L. Charles and L. Polterovich, Quantum speed limit vs. classical displacement energy, arXiv:1609.05395, Preprint, 2016.

[10] M. Entov, Quasi-morphisms and quasi-states in symplectic topology. In International Congress of Mathematicians, Seoul, 2014.

[11] M. Entov and L. Polterovich, Calabi quasimorphism and quantum homology. Int. Math. Res. Not. (2003), 1635-1676.

[12] M. Entov and L. Polterovich, Quasi-states and symplectic intersections. Comment. Math. Helv. 81 (2006), 75-99.

[13] M. Entov, L. Polterovich and F. Zapolsky, Quasi-morphisms and the Poisson bracket. Pure Appl. Math. Q. 3 (2007), 10371055.

[14] A. Floer, Symplectic fixed points and holomorphic spheres. Comm. Math. Phys. 120 (1989), 575-611.

[15] K. Fukaya, Y.-G. Oh, H. Ohta and K. Ono, Spectral invariants with bulk, quasimorphisms and Lagrangian Floer theory. arXiv:1105.5124, Preprint, 2011.

[16] L. Gilder, The Age of Entanglement: When Quantum Physics Was Reborn. Vintage Books USA, 2009.

[17] A. M. Gleason, Measures on the closed subspaces of a Hilbert space. J. Math. Mech. 6 (1957), 885-893.

[18] M. Gromov, Pseudoholomorphic curves in symplectic manifolds, Invent. Math. 82 (1985), 307-347.

[19] V. Guillemin, Star products on compact pre-quantizable symplectic manifolds. Lett. Math. Phys. 35 (1995), 85-89.

[20] L. Guth, The waist inequality in Gromov's work. In The Abel Prize 2008-2012, pp. 181-195.

[21] H. Hofer, On the topological properties of symplectic maps. Proc. Roy. Soc. Edinburgh Sect. A 115 (1990), 25-38.

[22] S. Ishikawa, Spectral invariants of distance functions. J. Topol. Anal. 8 (2016), 655-676.

[23] G. Kalai, The quantum computer puzzle. Notices Amer. Math. Soc. 63 (2016), 508-516.

[24] F. Lalonde and D. McDuff, The geometry of symplectic energy. Ann. of Math. 141 (1995), 349-371.

[25] X. Ma and G. Marinescu, Toeplitz operators on symplectic manifolds. J. Geom. Anal. 18 (2008), 565-611.

[26] L. Mandelstam and I. Tamm, The uncertainty relation between energy and time in nonrelativistic quantum mechanics. $J$. Phys.(USSR) 9, no. 249 (1945): 1.

[27] N. Margolus and L. B. Levitin, The maximum speed of dynamical evolution. Physica D: Nonlinear Phenomena 120 (1998), 188-195.

[28] D. McDuff and D. Salamon, J-holomorphic Curves and Symplectic Topology. Second edition, American Mathematical Society Colloquium Publications, 52, American Mathematical Society, Providence, RI, 2012.

[29] Y.-G. Oh, Floer cohomology of Lagrangian intersections and pseudo-holomorphic disks. II: $\left(\mathbb{C} P^{n}, \mathbb{R} P^{n}\right)$, Comm. Pure Appl. Math. 46 (1993), 995-1012.
[30] Y.-G. Oh, Construction of spectral invariants of Hamiltonian diffeomorphisms on general symplectic manifolds, in: The breadth of symplectic and Poisson geometry, Birkhäuser Boston, Inc., Boston, MA, 2005, pp. 525-570.

[31] Y.-G. Oh, Symplectic Topology and Floer Homology. Cambridge University Press, 2015.

[32] Y. Ostrover, Calabi quasi-morphisms for some non-monotone symplectic manifolds. Algebr. Geom. Topol. 6 (2006), 405434.

[33] A. Peres, Quantum Theory: Concepts and Methods. Fundamental Theories of Physics, 57, Kluwer Academic Publishers Group, Dordrecht, 1993.

[34] L. Polterovich, Symplectic geometry of quantum noise. Comm. Math. Phys., 327 (2014), 481-519.

[35] L. Polterovich, Symplectic rigidity and quantum mechanics, submitted to Proceedings of 7ECM, Berlin, 2016, available at https://sites.google.com/site/polterov/miscellaneoustexts/ symplectic-rigidity-and-quantum-mechanics.

[36] L. Polterovich and D. Rosen, Function Theory on Symplectic Manifolds. American Mathematical Society, 2014.

[37] M. Schlichenmaier, Berezin-Toeplitz quantization for compact Kähler manifolds. A review of results. Adv. Math. Phys. 2010, 927280

[38] M. Schwarz, On the action spectrum for closed symplectically aspherical manifolds, Pacific J. Math. 193 (2000), 419-461.

[39] S. Seyfaddini, Spectral killers and Poisson bracket invariants. J. Mod. Dyn. 9 (2015), 51-66.

[40] M. Usher, Deformed Hamiltonian Floer theory, capacity estimates and Calabi quasimorphisms. Geom. Topol. 15 (2011), 1313-1417.

[41] C. Viterbo, Symplectic topology as the geometry of generating functions, Math. Ann. 292 (1992), 685-710.

[42] J. von Neumann, Mathematical Foundations of Quantum Mechanics. Princeton University Press, Princeton, 1955. (Translation of Mathematische Grundlagen der Quantenmechanik, Springer, Berlin, 1932.)

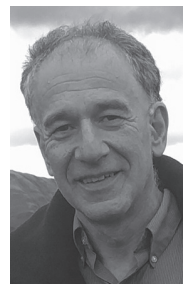

Leonid Polterovich [polterov@runbox.com] participated in Sinai's and Arnold's seminars at Moscow State University prior to his move to Israel in 1990 and received his $P h D$ degree from Tel Aviv University in 1990. Currently, he holds the Gordon Chair in Dynamical Systems and Symplectic Topology at Tel Aviv University and a visiting professorship at the University of Chicago. This article follows his plenary talk at the 7th European Congress of Mathematics in Berlin, 2016. 